\title{
Space-Charge Compensation in High-Intensity Proton Rings
}

\author{
A.V. Burov, G.W. Foster, V.D. Shiltsev \\ Fermi National Accelerator Laboratory \\ P.O. Box 500, Batavia, Illinois 60510
}

September 5, 2000

\begin{abstract}
Recently, it was proposed to use negatively charged electron beams for compensation of beambeam effects due to protons in the Tevatron collider [1]. We show that a similar compensation is possible in space-charge dominated low energy proton beams. The idea has a potential of severalfold increase of the FNAL Booster beam brightness. Best results will be obtained using three electron lenses around the machine circumference, using co-moving electron beam with time structure and profile approximately matched to the proton beam. This technique, if feasible, will be more cost effective than the straightforward alternative of increasing the energy of the injection linac.
\end{abstract}




\section{Introduction}

Defocusing repulsive forces due to self space charge lead to degradation of high-intensity particle beams. In circular accelerators, the figure of merit of such an effect is shift of incoherent betatron oscillations [2]:

$$
\Delta \nu_{S C}=-\frac{N_{t o t} r_{c} B_{f}}{4 \pi \varepsilon_{n} \beta_{p} \gamma_{p}^{2}},
$$

where $N_{\text {tot }}$ is total number of particles in the ring, $r_{c}=1.53 \cdot 10^{-18} \mathrm{~m}$ for protons, $\varepsilon_{n}$ is rms normalized emittance, $\beta_{p}=v_{p} / c$ and $\gamma_{p}$ are usual relativistic parameters, and the numerical factor $B_{f} \geq 1$ is defined by the ratio of the peak current to the average current. For the most of existing and planned high-brightness proton accelerators the tune shift lays in range of $-0.1 \ldots-0.5$ (see, e.g.,[3]). Above the threshold, coherent and incoherent instabilities result in the beam emittance dilution and particle losses which can not be tolerated.

The tune shift rapidly decreases with increase of the beam energy, thus, fast acceleration helps low-energy high-intensity proton machines. The short time at low energy is enough for developing only the lowest order resonances of incoherent motion (e.g., parametric resonance in a single particle motion inside the beam) as well as in coherent motion (e.g., quadrupole "breathing" modes of the beam shape variation).

As for incoherent motion, it should also be pointed out that in actual beams with non-uniform transverse beam distribution, the betatron oscillation frequency is a function of a particle's position in the beam. For example, for round Gaussian profile of proton density with rms size of $\sigma_{r}$ :

$$
n_{G}(r)=n_{0} \exp \left(-\frac{r^{2}}{2 \sigma_{r}^{2}}\right),
$$

the space charge tune shift is function of betatron amplitude $r$ :

$$
\Delta \nu(r)=\Delta \nu_{S C} \frac{4}{\alpha}[1-\exp (-\alpha / 4)] I_{0}(\alpha / 4),
$$

where $\alpha=\left(r / \sigma_{r}\right)^{2}$ and $I_{0}(x)$ is the modified Bessel function of order 0 . Thus, the tune shift for the beam core is larger than for the Gaussian tail. As a result, the core particles may encounter a resonance, while the outer particles remain unaffected. In principle, particle loss and emittance growth due to resonances can be reduced by proper choice of the betatron frequencies $\nu_{x, y}$ but it it possible only if the tune spread inside the core $\left(\simeq 1 / 3 \Delta \nu_{S C}\right.$ for $\left.r=2 \sigma_{r}\right)$ is less than the distance between resonances.

In bunched beams, transverse space charge force varies along the bunch proportionally to line charge density $\lambda(s)$, and, consequently, the tune shift depends on longitudinal position $s$ the way similar to Eq.(3) $\Delta \nu=\Delta \nu(r, s)$, i.e., again, the betatron frequency shift is smaller in the tails. As bunches of particles usually have much larger longitudinal dimension than transverse ones (typically $\sigma_{z} / \sigma_{r}>100$ ), the longitudinal space charge forces are weak, though visible as they distort RF potential. In this article we will not address the longitudinal space charge effects - it'll be a subject of separate consideration. 
Compensation of space charge forces on average, can significantly improve dynamics in lowenergy accelerators, and allow higher beam intensities.

\section{Compensation of Space Charge Forces in Proton Machines with Electron Beams}

Negatively charged electron beams can be used for compensation of space-charge effects in low energy proton beams the same way as in the ongoing Tevatron Electron Lens (TEL) project to compensate beam-beam effects due to protons in the Tevatron collider [1]. Protons going through the electron beam experience focusing force which has opposite sign to self space charge force and can precisely compensate the latter if:

1. transverse profile of the electron beam charge $n_{e}(r)$ is the same as proton beam profile,

2. temporal structure of the electron space charge force matches the proton space charge force,

3. integrated impact of electrons is equal to the total proton space charge impact over the ring.

Condition \#1 can be satisfied without difficulties - an electron beam profile can be controlled by special electrodes in the electron gun the same way as in the TEL [4]. The rigidity of the electron beam due to the solenoid field in the interaction region prevents the proton beam from distorting the electron beam. Moreover, exact matching of the transverse profiles of the electron and proton may not be necessary at all if the major brightness limitation comes not from incoherent (single-particle) motion but from coherent modes. In that case only linear forces count [5] and consequently, even rectangular electron beam current profile will help to stabilize the proton beam.

The time structure of a proton beam space charge force has two characteristic scales: one corresponding to beta-function variations with periods $T_{1}=L / \beta c$ (e.g., $L$ can be length of FODO structure half cell in strong focusing synchrotrons) and $T_{0}=C / \beta c$; the second one corresponds to the bunch structure $T_{2} \sim \sigma_{z} / \beta c$. It is impossible to have electron beam covering entire circumference of a proton accelerator. A more practical electron beam set-up should occupy only a small fraction of the ring circumference $C$. Therefore, the kick experienced by every proton has period $T_{0}$ but much shorter than the revolution period, and condition \#2 can not be be fulfilled for lattice period $T_{1}$ modulations. Nevertheless, the proton bunch structure can be matched by fast modulation of the electron current. Even having a short interaction region, there will be certain integrated compensation (on average) if condition \#3 is satisfied.

The condition \#3 of the compensation can be rewritten is the form of equality of the betatron tune shifts due to electron beam [1] and the proton space charge tune shift Eq.(1):

$$
\Delta \nu_{e} \equiv \frac{\beta_{r}}{4 \pi} \frac{\left(1-\beta_{e} \beta_{p}\right) J_{e} L r_{c}}{e \beta_{e} c \sigma_{e}^{2} \gamma_{p} \beta_{p}}=-\Delta \nu_{S C},
$$

where $J_{e}$ is electron current, $\sigma_{e}$ is the rms electron beam radius, $L$ is interaction length, $\beta_{r}$ is beta function at the location of the electron lens. The factor $1-\beta_{e} \beta_{p}$ reflects contributions of electric and 
magnetic forces due to co-moving electron beam. If the electron beam collides with proton beam, then the factor has to be changed to $1+\beta_{e} \beta_{p}$.

In fact, the necessity of fast longitudinal electron current modulation for matching the proton bunch profile almost definitely will require co-moving electron beam, i.e., the sign to be "-". Indeed, a characteristic modulation length which can be provided by an electron pulse interacting with protons along distance $L$ and moving in opposite directions (i.e., beams collide) is about $\sigma \simeq L\left(\beta_{p}+\right.$ $\left.\beta_{e}\right) / \beta_{e}$ (see Fig.8 in Ref.[1] for explanations). Therefore, even for $\beta_{e} \simeq \beta_{p}$ one gets $L \leq \sigma / 2$. That is not practical for short bunches as $\sigma \sim \sigma_{s} \sim 1 \mathrm{~m}$ because the interaction length has to be too short ( $L \leq 1 / 2 \mathrm{~m}$ ) and accordingly to Eq.(4), the required current becomes too large. On the other hand, if the electron and proton beams move in the same direction then $\sigma \simeq L\left(\beta_{p}-\beta_{e}\right) / \beta_{e}$ and one can provide very fast modulation if the electron velocity is close enough to the proton one $\beta_{e} \approx \beta_{p}$.

If one requires the rms size of electron beam has to match proton beam size in the location of the electron lens $\sigma_{e}^{2}=\varepsilon_{n} \beta_{p} \gamma_{p} \beta_{r}$, then we obtain the necessary electron current in the lens:

$$
J_{e}=J_{p} B_{f} \frac{C}{L} \frac{\beta_{e}}{\gamma_{p}^{2} \beta_{p}^{2}\left(1-\beta_{e} \beta_{p}\right)},
$$

As we will show in the next Section, optimum compensation requires even smaller current (about one third) than given by Eq.(5).

\section{Coherent Modes and Optimal Compensation}

The beam own space charge forces shift down both single-particle (incoherent) modes and the beam coherent modes. An exception is the coherent dipole mode (beam centroid motion) which does not depend on the space charge at all. If one of these modes is in resonance with the focusing lattice (structure resonance), the mode becomes unstable that leads to the beam loss and/or growth of the beam emittance. The electron lens shifts up all the frequencies, therefore, can be used compensation of the space charge effects. An important point is that the space charge and electron tune shifts are mode-specific, so if the electron lens compensates the space charge tune shift for one of the modes, it does not compensate it for others. Therefore, the electron lens can increase the proton beam brightness threshold but can not eliminate it. by the electron lens, but, at best, it could be increased. The tune shift introduced by the electron lens $\Delta \nu_{e}$ can be considered as proportional to the Laslett tune shift $\Delta \nu_{S C}$, i. e. $\Delta \nu_{e}=-\kappa \Delta \nu_{S C}$, with compensation coefficient $\kappa$ to optimize.

Within a simple model of the smooth approximation, assuming approximately equal vertical and horizontal lattice tunes $\nu_{x} \approx \nu_{y} \approx \nu_{0}$, and that the tune shifts are small in comparison with the tunes themselves $\Delta \nu<<\nu_{0}$, one gets single-particle frequency equal to

$$
\nu=\nu_{0}+(\kappa-1) \Delta \nu_{S C}
$$

Conventional stability analysis of the coherent motion is limited by a linear consideration of the dipole mode (first-order) and the envelope modes (second-order) [2]: coherent instabilities of higher orders do not reveal themselves neither experimentally, nor numerically; only integer and 
half-integer resonances are taken into account. The tune of the coherent dipole mode (frequency of the beam centroid oscillations) is not influenced by the space charge, so it goes as

$$
\nu_{d}=\nu_{0}+\kappa \Delta \nu_{S C}
$$

As for frequencies of beam envelope oscillations, there are two modes driven by linear forces. If vertical and horizontal lattice tunes are (approximately) equal, then for the symmetric mode, vertical and horizontal envelope oscillations are in phase so the beam "breathes" in both directions, while for the antisymmetric mode these phases are opposite. In the presence of the electron beam, the symmetric (+) and antisymmetric ( - ) coherent tunes can be calculated as

$$
\begin{aligned}
& \nu_{+}=2 \nu_{0}+(2 \kappa-1) \Delta \nu_{S C} \\
& \nu_{-}=2 \nu_{0}+(2 \kappa-3 / 2) \Delta \nu_{S C} .
\end{aligned}
$$

Dependence of the fractional tunes $(6,7,8)$ on the Laslett tune shift $\Delta \nu_{S C}$ without any compensation $\kappa=0$ is illustrated in Fig. 1 - result similar to Ref.[6]. One can see, that the system becomes unstable at threshold value of $\Delta \nu_{S C}=0.33$ when the antisymmetric mode tune becomes equal to integer $\Delta \nu_{-}=0$.

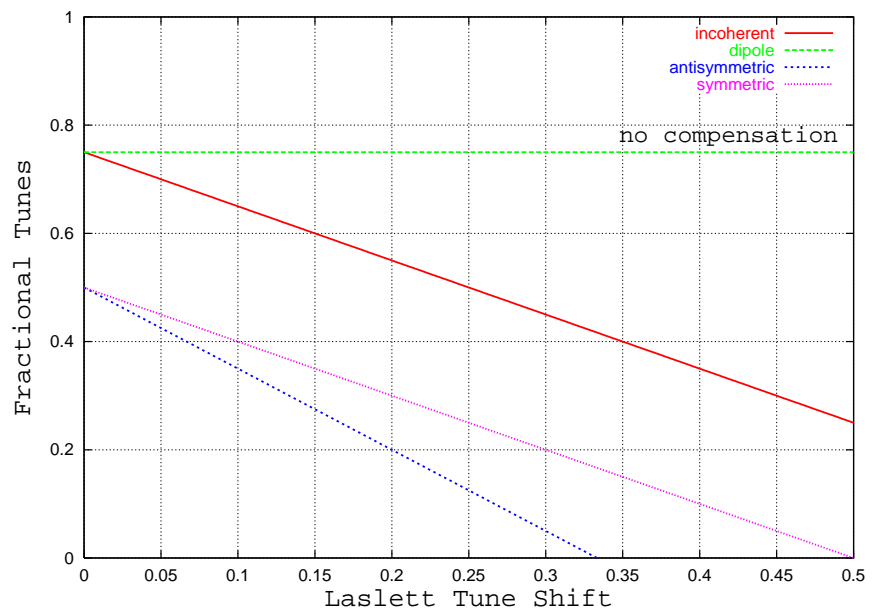

Figure 1: Incoherent, dipole and envelope fractional tunes versus Laslett tune shift. Compensation is not applied $\kappa=0$. The working point $(0.75,0.75)$ approximates the case of the Fermilab Booster.

Table 3 summarizes data from various space-charge limited low-energy proton synchrotrons [3] - the first row shows bare lattice tunes $\nu_{x}$ and $\nu_{y}$, the second row contains maximum empirically achieved Laslett tune shift for each machine $\Delta \nu$ exp. We compare experimental values with calculated distance $\Delta \nu_{\text {inc }}$ to the nearest incoherent resonance (third row) and the nearest coherent resonance $\Delta \nu_{\text {coh }}$.

The table reveals some noticeable features. First, the empirically reached Laslett tune shift sig-

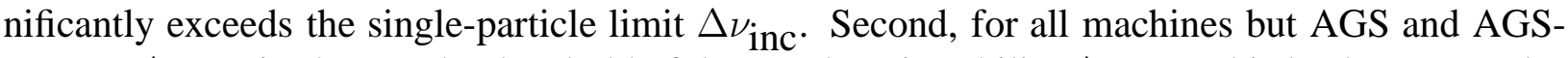
Booster $\Delta \nu_{\mathrm{exp}}$ is close to the threshold of the envelope instability $\Delta \nu_{\mathrm{coh}}$. This leads to a conclusion that the incoherent resonances may not be important; the actual threshold is rather determined by the coherent instabilities. Finally, we see that zero-current envelope tunes can be safely slightly 


\begin{tabular}{|l|c|c|c|c|c|r|r|}
\hline Parameter & KEK-B & FNAL-B & ISIS & AGS & AGS-B & CERN PS & CERN PS-2 \\
\hline$\nu_{x} / \nu_{y}$ & $2.17 / 2.3$ & $6.7 / 6.8$ & $3.7 / 4.2$ & $8.75 / 8.75$ & $4.8 / 4.9$ & $6.22 / 6.22$ & $6.22 / 6.28$ \\
\hline$\Delta \nu_{\exp }$ & 0.23 & 0.4 & 0.4 & 0.58 & 0.5 & 0.27 & 0.36 \\
\hline$\Delta \nu_{\text {inc }}$ & 0.17 & 0.2 & 0.2 & 0.25 & 0.3 & 0.22 & 0.22 \\
\hline$\Delta \nu_{\text {coh }}$ & $0.27 / 0.08$ & $0.36 / 0.08$ & 0.32 & 0.33 & $0.07 / 0.2$ & 0.27 & 0.33 \\
\hline
\end{tabular}

Table 1: Laslett tune shift reached empirically $\Delta \nu$ exp at various low-energy synchrotrons as compared with calculated Laslett tune shifts related to the nearest incoherent $\Delta \nu_{\text {inc }}$ and coherent $\Delta \nu_{\text {coh }}$ resonances.

above the resonance; the resonances at $\Delta \nu_{\text {coh }} \leq 0.1$ do not reveal themselves, perhaps, due to a weak space charge at the resonance crossing.

Behavior of the modes changes when the compensation is applied - see Fig.2. Dynamics of the dipole mode is rather different from the envelope modes, that makes it difficult to even double the threshold by varying the compensation degree $\kappa$, because one or another mode should cross linear resonance. Threshold Laslett tune shift of about 0.6 can be achieved with $\kappa=0.33$ as shown in Fig. 2 (a factor of $\approx 1.6$ in comparison with the no-compensation case).

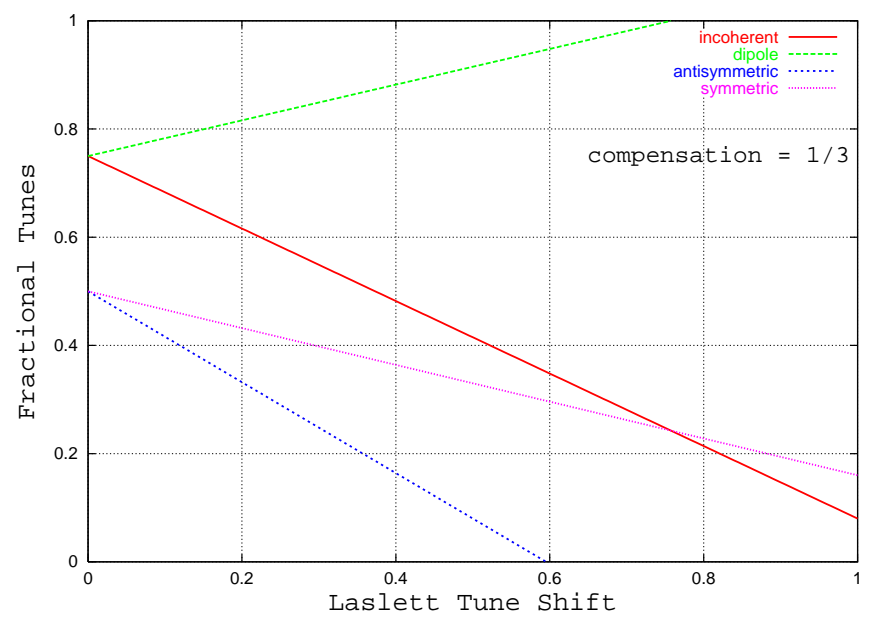

Figure 2: The same tunes for the compensation $\kappa=0.33$.

An additional option is associated with a possibility to have the degree of compensation $\kappa$ variable and dependent on the Laslett tune shift. In that case, modulation of the electron beam current is almost as easy as for the constant $\kappa$ strategy. The variable rate allows to jump over the resonances, for example, as illustrated in Fig. 3.

According to this plot, a simultaneous jump over two resonances $\left\{\nu_{d}\right\}=1$ and $\left\{\nu_{+}\right\}=0.5$ allows to reach the Laslett tune shift $\Delta \nu_{S C}=1.2$, more than 3 times higher than without compensation. However, this option has to be studied in more detail, in particular, finite width of the resonances have to be taken into account.

Symmetry of an accelerator focusing lattice is important for the space-charge compensation. If the lattice consists of $P$ identical periods, then strong and wide structure linear resonances occur 


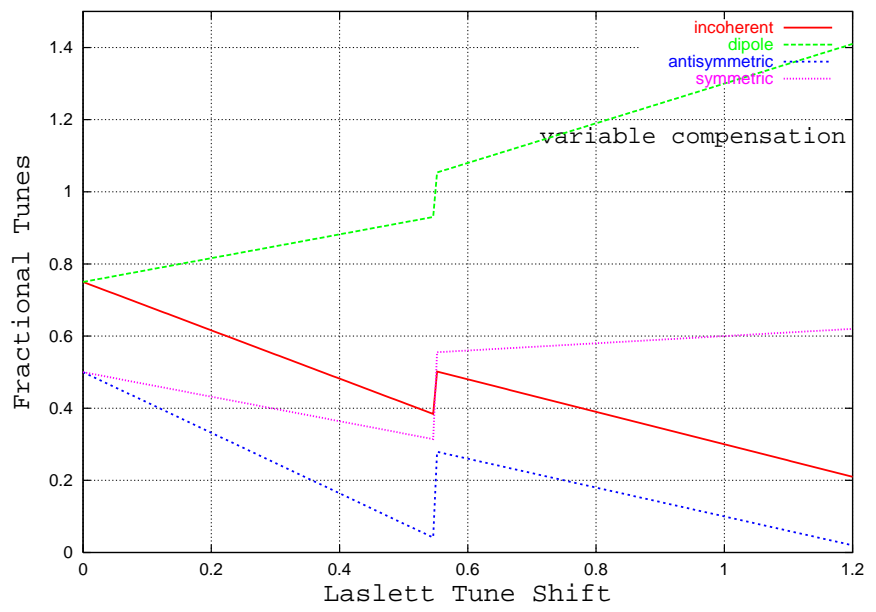

Figure 3: Tunes at jumped compensation: $\kappa=0.33$ for $\Delta \nu_{S C} \leq 0.55$, and $\kappa=0.55$ otherwise.

at coherent/incoherent frequencies $\nu_{\text {coh, inc }}=P m / 2$, where $m$ is integer. All other integer and half-integer tunes relate to relatively weak and narrow non-structure linear resonances, excited by periodicity perturbations (errors). Having high machine periodicity and properly chosen working point, one can have all the nearest linear incoherent and coherent resonances to be non-structure resonance, and thus allow, e.g., incoherent tune to cross half-integer or integer resonance, as in many machines analyzed above in Table 3.

A single strong electron lens may worsen the situation as it reduces the lattice periodicity from to $P$ to 1 . Thus, more than one compensation set-up is needed to keep nearest incoherent and coherent linear resonances being0 non-structure resonances. For example, the Fermilab Booster has periodicity $P=24$, and tunes $\nu_{x, y}=(6.7,6.8)$. If we have two electron lenses symmetrically placed in opposite parts of the ring, then the effective periodicity is reduced to $P=2$. Thus, the nearest incoherent resonance $\nu=6.5$ is non-structure (as it is without compensation devices at $P=24$ ), but the envelope resonances at $\nu_{-,+}=13=P m / 2=2 \cdot 13 / 2$ become structure. As the result, one has to have three identical compensators symmetrically positioned at the orbit in order to preserve non-structure weakness of the nearest incoherent and coherent resonances.

\section{Numerical Example: Fermilab Booster}

Let us consider the space-charge compensation in the Fermilab Booster. Parameters of FNAL Booster are presented in Table $1[7,3])$. Accordingly to Eq.(1) the space charge tune shift at injection is about

$$
\Delta \nu_{S C}(t)=-0.435 \cdot B_{f}(t) \cdot\left(\left[\varepsilon_{n} \beta_{p} \gamma_{p}^{2}\right](t) /\left[\varepsilon_{n} \beta_{p} \gamma_{p}^{2}\right](t=0)\right)
$$

if parameters are $N_{t o t}=5 \cdot 10^{12}, \varepsilon_{n}=1 \pi \mathrm{mm} \cdot \operatorname{mrad}, \gamma_{p}=1.4, \beta_{p}=0.7$. The period of strong space-charge action is comparatively short - injection itself takes about 12 turns, then some 20-40 turns the beam circulates without RF to wipe off the Linac RF structure - it corresponds to $B_{f}=1$. After that the Booster RF is being adiabatically turned on over some 100 turns, and then the beam energy rapidly grows, e.g., to $2.5 \mathrm{GeV}$ after about 3000 turns. Note, that the ratio of peak to average 
current rapidly increases from 1 to $B_{f}(t) \approx 2-3$ over a hundred turns, while the factor $1 /\left(\beta_{p} \gamma_{p}^{2}\right)$ slowly decreases 4 times from 0.71 to 0.174 over thousands of turns. If there would be no particle loss and emittance blow up then the Booster beam space charge tune shift parameter would reach the maximum value of right after bunching $\Delta \nu_{S C} \approx 0.9-1.3$.

Table 2: FNAL Booster Parameter List

\begin{tabular}{|ll|r|}
\hline Parameter & & \\
\hline Circumference, & $\mathrm{m}$ & 474 \\
Proton energy at injection, & $E_{\text {inj }}, \mathrm{GeV}$ & 0.4 \\
Peak energy, & $E_{\text {max }}, \mathrm{GeV}$ & 8.0 \\
Cycle time, & $T_{c}, \mathrm{~ms}$ & 67 \\
Ramp time, & $T_{\text {ramp }}, \mathrm{ms}$ & 33 \\
Number of protons, & $N_{\text {tot }}$ Run Ib at $2.5 \mathrm{~Hz}$ & $3 \cdot 10^{12}$ \\
& $N_{t o t}$ Run IIa at $0.7 \mathrm{~Hz}$ & $5 \cdot 10^{12}$ \\
Number of bunches, & $N_{\text {tot }}$ Run IIb at $8 \mathrm{~Hz}$ & $5 \cdot 10^{12}$ \\
RF harmonics, & $N_{b}$ & 83 \\
RF frequency, & $h_{R F}$ & 84 \\
Tunes, & $f_{R F}, \mathrm{MHz}$ & $38-53$ \\
Transition $\gamma_{t}$ & $\nu_{x}, \nu_{y}$ & $6.7 / 6.8$ \\
Beta-function, & & 5.4 \\
Dispersion function, & $\beta_{\text {min,avg,max }}, \mathrm{m}$ & $6.0,11.3,20.5$ \\
Transverse emittance at injection, & $\varepsilon_{n}, \mathrm{~mm}$ mrad, rms & -1.9 \\
Transverse emittance at extraction, & $\varepsilon_{n}^{\text {extr }} \mathrm{mm}$ mrad, rms & 1 \\
Efficiency, & & 3 \\
\hline
\end{tabular}

Currently, due to space charge effects, the beam loses some $35-40 \%$ of particles during that initial period of 5-6 ms, and its transverse emittance blows up about 3 times [8]. From that we can conclude that the beam brightness deteriorates quickly if the space charge tune shift exceeds $\Delta \nu_{S C} \geq-(0.25-0.4)$, that is consistent with conclusions of previous studies in the Booster [9].

According to our analysis in the previous Section, the optimal compensation coefficient is less than $1, \kappa=0.33-0.55$, therefore, we can rewrite Eq.(5) to get the peak electron current to compensate the space charge effects at the current Booster beam intensity of $J_{\max }=J_{p} B_{f}=0.35[A] B_{f}$ : 


$$
J_{e}=0.35[A] B_{f} \cdot \kappa \cdot\left(\beta_{e}\left(1-0.7 \beta_{e}\right)\right) \cdot\left(474[m] / L_{t o t}[m]\right),
$$

where we took into account that $\gamma_{p} \beta_{p} \approx 1$ at injection. As concluded in Section 3 above, it is beneficial to install three equally strong lenses in the Booster, so if each is $L=4 \mathrm{~m}$ long (to fit $6 \mathrm{~m}$ long drift sections), then $L_{t o t}=3 \times L=12 \mathrm{~m}$ and for the $B_{f}=3$ we yield $J_{e}(\kappa=0.33)=12.7 \mathrm{~A}$ of the peak current in each of three $80 \mathrm{kV}$ electron beams, $\beta_{e}=0.56$. The choice of the electron beam energy and $\beta_{e}$ is made to satisfy condition $\sigma_{s} \approx L\left(\beta_{p}-\beta_{e}\right) / \beta_{e}$, where the rms proton bunch length of $\sigma_{s}=1 \mathrm{~m}$ is taken. Again, if the goal is to deliver the existing intensity in significantly smaller emittance (i.e., prevent the emittance blow-up), then the electron current could be $12.7 \mathrm{~A}$.

If the ultimate goal is to double the Booster intensity - that is allowed by the FNAL Linac and will require more Booster RF power - then $J_{e}(\kappa=0.33)=25.4$ A of the electron current are needed. We will use that maximum number in our further calculations.

In general, the Booster Electron Lens (BEL) will be much alike the Tevatron Electron Lens (TEL) for compensation of beam-beam effects - see Fig.4. All considerations of the TEL [1] are applicable to the BEL, and we present here only brief summary of technically important features of the set-up.

The BEL will consist of three solenoid magnets, electron gun and collector, high voltage modulator, vacuum system, beam diagnostics and control.

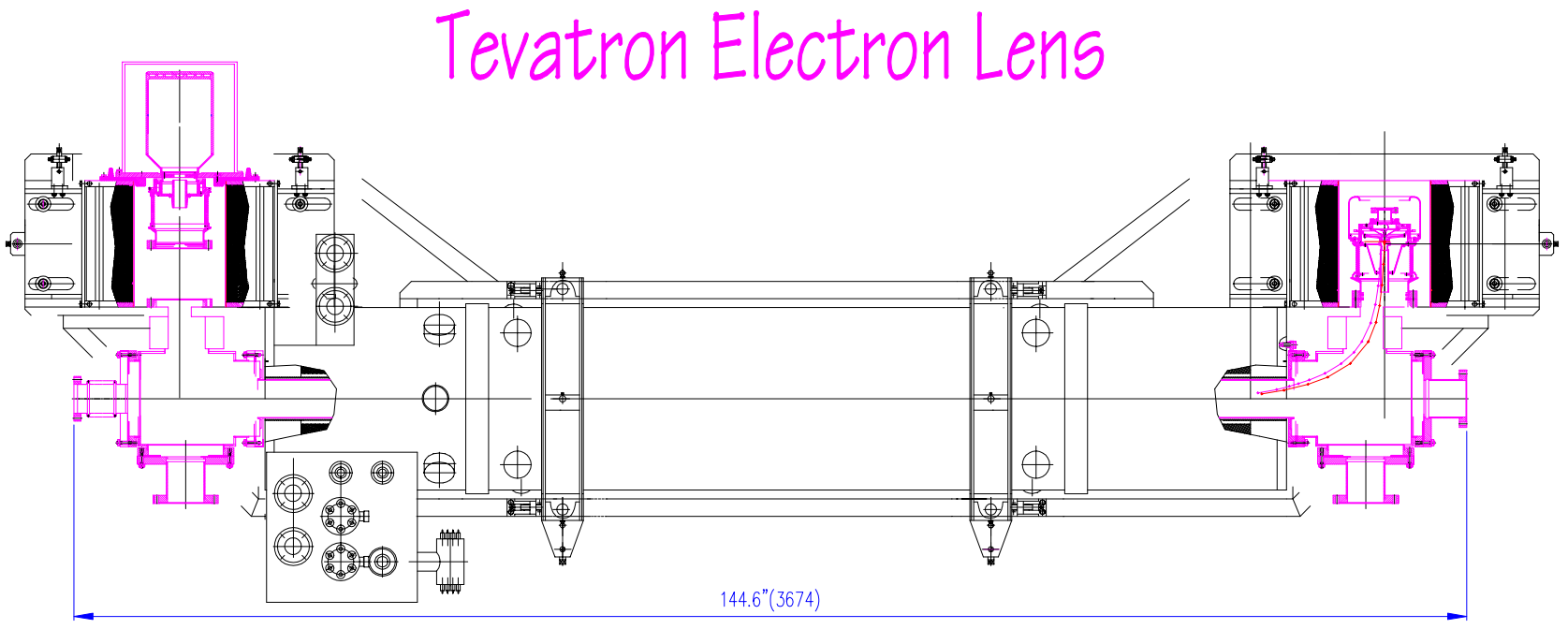

Figure 4: Layout of the Tevatron Electron Lens.

The main $4 \mathrm{~m}$ long normal-conducting solenoid magnet will provide up to $B=15 \mathrm{kG}$ of longitudinal magnetic field. Magnetic field lines in that magnet has to be straight within $1 \mathrm{~mm}$ over $4 \mathrm{~m}$ length. 4-8 dipole corrector coils will help to make the field lines straight as well as be used for the electron beam steering. $B_{g}=3-4 \mathrm{kG}$ side solenoids are necessary for electron beam injection and extraction. It may be necessary to add two toroids to make that 90 degrees turns smoother. This will be the subject of more detailed studies.

Electron gun with high perveance $\mathcal{P}=3 \mu \mathrm{A} / \mathrm{V}^{3 / 2}$ is some modification of the TEL electron gun [4]. It has an oxide cathode with 1-1.6 inch diameter, and can provide $J_{e}=\mathcal{P} U_{a}^{3 / 2}=25.4 \mathrm{~A}$ of current if the voltage between anode and cathode is $U_{a}=41.5 \mathrm{kV}$. Proper choice of the gun 
geometry allows to get the beam with bell-shape or close to to Gaussian current profile with rms size at the cathode of about $\sigma_{c}=13 \mathrm{~mm}$. The gun is immersed into the magnetic field $B_{g}$ which keeps electron beam from from spreading due to its own space charge forces. The electron beam transverse size is later adiabatically compressed to about $8 \mathrm{~mm}$ as the beam enters higher field of the main solenoid (the value of $\sigma^{2} B$ is adiabatic invariant of the beam).

It can be shown that impedance of the electron beam is negligible if the magnetic field in main solenoid is more than $B>4 \mathrm{kG}$.

After symmetric 90 degree turn, the beam goes into an electron collector with $\mu \mathcal{P} \approx 10$, similar to the one already built for the TEL. The collector absorbs more than $99.5 \%$ of the beam current. The collector can be placed under potential higher than the cathode potential, so beam energy recirculation is possible. Average power dissipated in each of three electron beam collectors $W_{\text {coll }}=50 \mathrm{~kW}$ - under the assumption of $75 \%$ power recirculation efficiency, $6.6 \mathrm{~ms}$ pulse duration and $15 \mathrm{~Hz}$ repetition rate of injection into Booster.

Depending on the longitudinal proton bunch profile, we will need or will not need $38 \mathrm{MHz}$ electron current modulator. If the longitudinal bunch profile (current) is flat, than no modulator is needed. If the bunch shape is more like Gaussian, then we have to modulate some $40 \mathrm{kV}$ of the cathodeanode voltage. That is about $5 \mathrm{MW}$ peak RF power at $38 \mathrm{MHz}$, and will require about $50 \mathrm{~kW}$ (pulsed) RF generator for a narrow band RF system with $Q \sim 100$.

Vacuum system of the BEL has to provide vacuum better than $10^{-8}$ Torr in order to avoid significant ion production (ions can cause electron beam instability in the main solenoid).

A compensating solenoid magnet may need to be required to prevent strong coupling due to field of main solenoid.

Finally, we list main parameters of the Booster Electron Lenses for two scenarios: an Emittance Upgrade scenario where the goal is avoiding 3 fold emittance blow-up at existing intensities, and a Double Intensity upgrade which requires higher electron beam current:

\section{Emittance Upgrade Double Intensity}

$\begin{array}{lcc}\text { maximum e-current } J_{e}, \mathrm{~A} & 12.7 & 25.4 \\ \text { e-beam length } & 3 \text { lenses, each } L=4 \mathrm{~m} \text { long } & \text { 3 lenses, each } L=4 \mathrm{~m} \text { long } \\ \text { rms e-beam size, } \sigma_{e}, \mathrm{~mm} & 4.5 & 8 \\ \text { cathode radius, mm } & 12 & 20 \\ \text { B-field in gun/main solenoid, } \mathrm{kG} & 3 / 11 & 4 / 13 \\ \text { e-beam energy } U_{e}, \mathrm{kV} & 80 \mathrm{kV} & 80 \mathrm{kV} \\ \text { anode-cathode voltage } U_{a}, \mathrm{kV} & 26 & 41 \\ \text { HV RF modulator power, } \mathrm{kW} & 20 & 50\end{array}$

\section{Discussion and conclusions}

The use of low-energy high-current electron lenses to compensate space charge effects in highbrightness proton accelerators looks very promising. Our consideration shows that in optimal configuration few (three) electron lenses can compensate space charge tune shifts as large as $\Delta \nu_{S C} \approx$ 
$0.6-1.2$. With further increase of the proton beam brightness some coherent modes or incoherent motion can become unstable while crossing particular resonances. Depending on working point and $\Delta \nu_{S C}$ the electron beam profile should or should not approximately match the transverse profile of the proton beam. For example, if the space charge coherent modes limit the accelerator performance, than there is not need in a perfect profile matching. Still, matching of longitudinal proton bunch profile seems to be necessary. For that the electron beam current need to be correspondingly modulated.

We have considered electron lenses for the compensation in the Fermilab Booster. We found that electron beam systems are quite feasible for realization of either proton beam emittance upgrade or two-fold intensity upgrade in the Booster. These upgrades can significantly improve the performance of fixed target experiments, e.g., NuMI; and/or will allow to increase the antiproton production, proton beam brightness in collision and ultimately increase the luminosity of the Tevatron.

We note that neutralization or compensation of a beam space charge was the subject of many theoretical and experimental studies in the past. Notable examples are Budker relativistic stabilized electron beam [10], and neutralization of electron beam by ions in plasma [11], [12]. Bibliography on the topic and consideration of charge neutralization effects including instabilities can be found in the book of M. Reiser [2]. Recently, it was proposed in Ref.[13] to use highly non-linear external focusing to conserve emittance of a high-brightness beam. The nonlinearities are assumed to be created by a plasma lens with a specific distribution of particles or by AG quadrupole structure with a higher mode (duodecapole) field component. Both proposals are shown to work well in simulations but far from being practical for existing rings and their feasibility is not being considered. In addition, the dynamics of the coherent modes has not been considered at all, while thought to be the most important phenomena in the space-charge dominated beams [5], [2].

In principle, there are other tools to compensate the tune shift but they all have serious disadvantages. For example, pulsed $\gamma_{t}$-jump quadrupoles in the Booster can shift the tune for about 2, but it does not seem possible to modulate their gradients over the bunch length of few ns. RF quadrupoles can provide necessary modulation along the bunch but a) can not match transverse beam profile; and b) will require very powerful RF sources.

The proposed electron lens has a number of advantages: easier beam shape control and current modulation, moderate power consumption. The parameters of the lens are quite feasible that make the proposal attractive for further consideration and practical implementation.

\section{Acknowledgments}

We acknowledge helpful discussions with R.Webber, J.Lackey, J.Marriner and S.Holmes.

\section{References}

[1] V. Shiltsev, V. Danilov, D. Finley, A. Sery, "Compensation of Beam-Beam Effects in the Tevatron with Electron Beams”, Phys.Rev. ST Accel. Beams, 2, 071001 (1999).

[2] M. Reiser, Theory and Design of Charged Particles Beams, John Wiley\&Sons (1994). 
[3] W. Chou, "High Intensity High Brightness Proton Machine Tables", ICFA Beam Dynamics Newsletter, No.20 (Aug.1999).

[4] A. Shemyakin, et.al, "Performance of a High-Perveance Electron Gun with a Convex Cathode”, Proc. EPAC'00, Vienna (2000).

[5] F. Sacherer, "RMS Envelope Equations with Space Charge”, IEEE-NS 18 (1971), p.1105.

[6] R. Baartman, "Betatron Resonances with Space Charge", in it Proc. Int. Workshop on Particle Dynamics in Accelerators, Tsukuba, Japan (1994), KEK Proceedings 95-7 (1995), p.273.

[7] E.L. Habbard, “Booster Synchrotron”, FNAL TM-405 (1973).

[8] R. Webber, FNAL-Conf-00/193 (Aug.2000); and R. Webber, private communication.

[9] C. Ankenbrandt, S. Holmes, "Limits on Transverse Phase Space Density in the Fermilab Booster", Proc. 1987 IEE PAC, Washington, D.C., v.2, p.1066 (1987);

S. Holmes, "Critical Beam Intensity Issues in Hadron Colliders", in AIP Conf. Proc. 377 on Space Charge Dominated Beams and Application of High Brightness Beams, Bloomington, IN (1995), p.42.

[10] G. Budker, "Relativistic Stabilized Electron Beam", CERN Symposium on High Energy Accelerators, CERN, Geneva (1956); Sov. Atomnaya Energiya (1979).

[11] M. Nezlin, Physics of Intense Beams in Plazmas, Institute of Physics Pub (1993).

[12] A.V. Burov, V.I. Kudelainen, V.A. Lebedev, V.V. Parkhomchuk, A.A. Sery, and V.D. Shiltsev, "Experimental Investigation of an Electron Beam in Compensated State", Preprint INP 89116, Novosibirsk (1989), Preprint CERN/PS 93-03 (AR), CERN, Switzerland (1993).

[13] Yu. Batygin, "Conservation of High Current Beam Emittance in a Nonlinear Focusing Field", Phys.Rev.E , 53, p.5358 (1996) 